Recepción: 15 / 04 / 2017

Ciencias Financieras

Aceptación: 01 / 05 / 2017

Artículo Científico

Publicación: 15 / 05 / 2017

\title{
Construyendo una cultura financiera caso
}

\author{
Building a financial culture case
}

\section{Construindo um evento de cultura financeira}

Roberth F. Ramirez-Granda roberth.ramirezgr@ug.edu.ec

Milton I. Manosalvas-Tola" milton.manosalvasto@ug.edu.ec

Antonio F. Bazurto-Quiroz ${ }^{\mathrm{m}}$ antoniobasurtoq@ug.edu.ec

Correspondencia: roberth.ramirezgr@ug.edu.ec 


\section{Resumen}

Por lo general las economías estables y vigorosas cuentan con mercados de valores desarrollados y en donde sus habitantes ven a estos como su mejor opción de financiamiento, sin embargo en nuestro país el uso de estos mercados es deficiente, ocasionado básicamente por la deficiente cultura en temas bursátiles. En el Ecuador la creación de la bolsa de valores se realizó con el objetivo de transformar los procesos comerciales y económicos ofreciendo diferentes productos, mejores plazos y oportunidades de diversificación, pese a ello este no ha tenido la acogida esperada, razón por la cual se realizó la presente investigación con el propósito de determinar la influencia que tiene la inversión en la bolsa de valores ecuatoriana en el crecimiento económico de la provincia del Guayas, en este estudio luego de la sistematización del problema, definición de objetivos y justificación, se revisó el marco teórico, observando aspectos relacionados con la reducción de los niveles de intermediación en la colocación de capitales, lo que implica disminución de los niveles de tasas de interés como un elemento dinamizador de la economía. Se planteó una metodología que nos permitió recabar información con el fin de validar las hipótesis del estudio. Una vez obtenidos los resultados del proceso investigativo se optó por diseñar un instructivo básico sobre temas de inversión y financiamiento bursátil con el cual se pretende lograr en los habitantes de la provincia mayor nivel de conocimientos que a su vez permita incrementar los índices de inversión bursátil y en conclusión lograr crecimiento de la economía y del bienestar población en general (crecimiento y desarrollo económico).

Palabras clave: Mercado de valores; cultura bursátil; crecimiento económico; inversión; financiamiento. 


\begin{abstract}
Stable and vigorous economies usually have developed securities markets and where their inhabitants see them as their best financing option, however in our country the use of these markets is deficient, caused basically by the deficient culture in subjects Stock exchanges. In Ecuador, the stock exchange was created with the objective of transforming commercial and economic processes by offering different products, better terms and diversification opportunities, despite the fact that it has not had the expected reception, which is why The present investigation with the purpose of determining the influence that the investment in the Ecuadorian stock exchange has on the economic growth of the province of Guayas, in this study after the systematization of the problem, definition of objectives and justification, the framework was revised Observing aspects related to the reduction of the levels of intermediation in the placement of capital, which implies a reduction of interest rate levels as a dynamizing element of the economy. A methodology was developed that allowed us to gather information in order to validate the hypotheses of the study. Once obtained the results of the investigative process, it was decided to design a basic instruction on investment and stock market financing issues, which seeks to achieve in the inhabitants of the province a higher level of knowledge that in turn allows to increase the stock market indexes and In conclusion achieve growth of the economy and the general population welfare (growth and economic development).
\end{abstract}

Key words: Stock market; stock exchange culture; economic growth; investment; financing. 


\section{Resumo}

Economias geralmente estáveis e vigorosas desenvolveram mercados de valores mobiliários e onde as pessoas vêem estes como a melhor opção de financiamento, no entanto, em nosso país, o uso desses mercados é pobre, causada principalmente por questões de cultura pobres estoque. No Equador, a criação da bolsa de valores foi realizada com o objectivo de transformar os processos comerciais e económicas que oferecem produtos diferentes, melhores condições e oportunidades de diversificação, no entanto, esta não teve o acolhimento esperado, por que foi levada a cabo esta investigação, a fim de determinar a influência do investimento em valores equatorianos ações no crescimento económico da província de Guayas, neste estudo, após a sistematização do problema, definindo objetivos e fundamentos, o quadro foi revisto aspectos teóricos observando redução dos níveis de intermediação na colocação de capital, o que significa diminuição dos níveis de taxas de juros como estímulo para a economia. uma metodologia que nos permitiu reunir informações a fim de validar a hipótese do estudo foi levantado. Depois de obter os resultados do processo de pesquisa escolheu para projetar uma instrução básica sobre questões de investimento e de financiamento que deve ser alcançado entre os habitantes da província maior nível de conhecimento que por sua vez permite aumento das taxas de títulos de investimento e em conclusão crescimento económico e população em geral bem-estar (crescimento e desenvolvimento económico).

Palavras chave: Mercado de valores; cultura bursátil; crescimento económico; inversão; financiamento 


\section{Introducción.}

El tema de inversión en la bolsa de valores ecuatoriana da la impresión de que es un tema dirigido tan solo a las grandes empresas, lo que en realidad no es así, puesto que concierne a todas aquellas personas que generen excedentes económicos y que deseen obtener mayor rentabilidad por sus inversiones, cualquiera que esta sea (renta fija o renta variable) y también a quienes necesitan recursos con tasas de interés menores a las que ofrece el mercado financiero convencional. (Díaz Mata, 2005) (Pico Pico \& Pulgar León, 2006)

Se ha determinado que en la provincia del Guayas existen los recursos económicos, pero sin embargo por la información asimétrica que se maneja por parte del mercado de valores, no ha permitido a sus habitantes tener conocimientos sobre financiamiento e inversión bursátil llevando a la gran mayoría de ellos a creer que como prestamistas o prestatarios potenciales solo tendrán oportunidades dentro del sistema bancario tradicional, este es un hecho en la vida para muchos de nosotros. El objetivo del presente trabajo de investigación es que contribuya a despertar en los lectores un mayor entusiasmo y motivación para utilizar nuevos medios de financiamiento e inversión, fomentando el ahorro interno e impulsando la distribución de los recursos hacia actividades productivas que beneficien a la población en general. (Moreno García, Vázquez Cotera, Hernández Mejía, \& Larios Ojeda, 2015) (Paresh Kumar, Sagarika, \& Seema, 2011) (Peña, 1991)

En cuanto a la metodología se realizó un estudio de campo, en el cual se tomaron en cuenta aspectos tanto cualitativos como cuantitativos de la población de la provincia del Guayas, caracterizando la misma con personas mayores de edad hasta los 70 años aproximadamente, y luego de haber aplicado un tipo de muestra no probabilística nos dio como resultado la aplicación de 384 encuestas además de 3 entrevistas realizadas a personas que conocen la temática estudiada, la 
propuesta no solo consiste en diseñar un folleto con largas listas de conceptos, sino más bien se plantea sugerencias específicas y detalladas acerca de cómo aprovechar mejor las oportunidades expresadas en: tasas, estructura de capital, plazos y productos que ofrece este mercado. Es decir el principal propósito es que los usuarios puedan comprender que es posible financiar sus proyectos y convertirse en tenedores de títulos en lugar de ser depositantes, si conocen y saben utilizar correctamente los mercados de valores. En síntesis lo que se va a diseñar es un instructivo que pueda constituirse como una de las mejores guías hacia la toma de decisiones de inversión en los mercados de valores, para la población de la provincia del Guayas. (Garay Anaya, 2015)

El presente estudio pretende determinar el nivel de inversión en la bolsa de valores ecuatoriana por parte de los habitantes de la provincia del Guayas a través de una investigación de tipo científica que permita determinar su incidencia en el desarrollo socioeconómico.

\section{Materiales y métodos.}

En el presente trabajo se realizara un estudio de campo puesto que se necesitara interactuar con las fuentes hacer consultadas puesto que de ellas se obtendrá datos relevantes que serán de importancia para llevar a cabo la presente investigación.

Por definición algunos de los aspectos relacionados al tipo y diseño de la investigación son los siguientes:

Considero que la Investigación es de tipo explicativo ya que nos permitirá mostrar los resultados obtenidos verificar e interpretar la validez de las hipótesis planteadas. Así mismo se plantea de tipo descriptivo ya que con la aplicación de este tipo de investigación se buscara ofrecer una descripción del problema a través del análisis de las variables involucradas. También se 
considera de tipo correlacional en razón de a través del análisis causa efecto podemos mostrar la relación entre las variables es decir el grado en que influye el nivel de inversión en la bolsa de valores en el crecimiento socioeconómico de la provincia del Guayas.

Puedo señalar además que la Investigación es cuantitativa/cualitativa debido a que se analizan variables cuantitativas más aun cuando nos referimos a que existe desconocimiento nos referimos a comportamientos sociales y culturales que son elementos cualitativos, en tal sentido tomaremos aspectos cualitativos que nos permiten deducir temas cualitativos será cuantitativa, puesto que las variables que hemos considerado serán medidas de acuerdo a la información que nos proporciones los datos obtenidos, lo cual nos conduce también al método general hipotético deductivo.

En la región costa de la República del Ecuador se encuentra ubicada la provincia del Guayas la cual está habitada por más del $30 \%$ de la población Ecuatoriana según datos del instituto de estadísticas y censo realizado en el año 2010 cuenta con una población de 1.829.569 mujeres y 1.815.914 hombres el cual nos da un total de 3.645.483 habitantes. Guayas es la provincia más importante del Ecuador puesto que es la que más aporta al producto interno bruto según el último estudio del banco central del Ecuador Guayas con un 26, 44\% su economía se basa fundamentalmente en "la agricultura ganadería y pesca también la industria manufacturera los productos de mar y el puerto marítimo de Guayaquil son ejes importantes de la economía.

\section{Proceso de selección}

Para llevar a cabo el proceso de selección de los individuos se realizara de forma aleatoria simple, de forma que todos los elementos de la población a ser estudiadas tendrán la misma 
probabilidad de ser seleccionados para la muestra que permita llevar a feliz término el proceso de selección concerniente a la problemática que se ha planteado.

Los métodos y las técnicas

En el diseño de la presente investigación se utilizara los diferentes métodos y técnicas, con el objetivo de alcanzar datos certeros y confiables.

\section{Métodos empíricos}

Deductivo.-Se utilizara el método deductivo porque la investigación buscara inferir en los datos tomando como referencia una teoría básica general.

Analítico - Sintético.-En la presente investigación utilizaremos este método puesto que nos permitirá llegar a verdad, para ello procederemos a dividir los elementos que intervienen en nuestra investigación, para reunir nuevamente los elementos que sean lógicos e interactúen entre si "hasta completar y demostrar la verdad del conocimiento. Hay quienes lo manejan como métodos independientes".

\section{Técnicas de investigación}

Encuesta: A través de esta técnica se recaudara datos por medio de un conjunto de preguntas para obtener opiniones de tipo empírico esto se hará por medio de un conjunto de preguntas al cual se conoce como cuestionario.

Entrevista: esta técnica permitirá obtener información de tipo científico por parte de las personas entrevistadas la cuales profundizaran más en el tema debido al mayor conocimiento que poseen sobre la problemática a tratar. 
Tratamiento estadístico de la información

La información y datos obtenidos a través de las encuestas aplicadas, serán tabulados, interpretados y analizados a través de gráficos estadísticos, las entrevistas realizadas también, serán interpretadas de forma general puesto que la información obtenida es de cualitativa.

Con las respuestas obtenidas en la primera pregunta de la encuesta realizada se busca establecer el nivel conocimiento que tienen los habitantes de la provincia del Guayas sobre inversión en la bolsa de valores ecuatoriana.

\section{Resultados.}

\section{Análisis de la situación actual}

Considero que las técnicas e instrumentos utilizados en la presente investigación (entrevistas y encuestas) las cuales fueron dirigidas a docentes, PYMES, y profesionales involucrados en temas de inversión en bolsa de valores ecuatoriana, ha permitido obtener información fundamental que será de gran ayuda para sustentar y defender mi propuesta.

Análisis comparativo, evolución, tendencia, y perspectivas

\section{¿Qué conocimientos tiene usted sobre inversión en bolsa de valores?}

\begin{tabular}{ccc} 
ALTERNATIVAS & \# ENCUESTADOS & PORCENTAJES \\
\hline MUCHO & 45 & $12 \%$ \\
POCO & 53 & $14 \%$ \\
NADA & 286 & $74 \%$
\end{tabular}

Tabla $N^{\circ}$ 1.- Nivel de conocimientos con respecto a la importancia de inversión en la bolsa de valores ecuatoriana 
Análisis

Observamos que 7 de cada 10 individuos No tienen conocimientos sobre inversión en la bolsa de valores ecuatoriana, las alternativas Poco y Nada para esta pregunta corresponden al $1.4 \%$ y $1.2 \%$, por lo tanto son mínimas para el análisis.

Interpretación

De la información obtenida se puede interpretar que el $74 \%$ de los encuestados no tiene mayores conocimientos sobre inversión en bolsa de valores.

¿Considera usted que la inversión en los mercados de capitales es un factor clave para generar un crecimiento económico alto?

\begin{tabular}{ccc} 
ALTERNATIVAS & \# ENCUESTADOS & PORCENTAJES \\
\hline TOTALMENTE DE & 180 & $47 \%$ \\
ACUERDO & & $53 \%$ \\
DE ACUERDO & 204 & $0 \%$ \\
EN DESACUERDO & 0 &
\end{tabular}

Tabla $N^{\circ}$ 2.- Nivel de conocimientos con respecto a la importancia de inversión en mercados de capitales para generar un crecimiento económico alto

Análisis

Observamos que 5 de cada 10 individuos está De acuerdo, mientras que los otros 5 están Totalmente de acuerdo. La alternativa En desacuerdo para esta pregunta corresponde al 0\%, por lo tanto no será de importancia para el análisis. 
Interpretación

De la información recabada podemos interpretar que $43 \%$ de los encuestados está Totalmente de acuerdo mientras que el 53\% está De acuerdo con que un mayor nivel de inversión en el mercado de valores puede generar un alto crecimiento económico.

¿Cree usted que la inversión en instituciones financieras convencionales genera mayor rentabilidad que la inversión en la bolsa de valores?

\begin{tabular}{ccc} 
ALTERNATIVAS & \# ENCUESTADOS & PORCENTAJES \\
\hline TOTALMENTE DE & 17 & $4 \%$ \\
ACUERDO & & $29 \%$ \\
DE ACUERDO & 111 & $67 \%$ \\
EN DESACUERDO & 257 &
\end{tabular}

Tabla $N^{\circ}$ 3.- Nivel de conocimientos con respecto a la a la rentabilidad que genera invertir en instituciones financieras convencionales $o$ en bolsa de valores

Análisis

Observamos que 7 de cada 10 individuos está en Desacuerdo mientras que 3 están De acuerdo. La alternativa Totalmente de acuerdo para esta pregunta corresponde al $0,4 \%$, por lo tanto es insignificante para el análisis.

Interpretación

De los resultados obtenidos se puede interpretar que el $67 \%$ de los individuos reconoce que la inversión en la bolsa de valores ecuatoriana no genera mayor rentabilidad que la inversión en bolsa de valores. 
Según su opinión. Las empresas no utilizan los valores negociables para financiar sus actividades a causa de:

\begin{tabular}{ccc} 
ALTERNATIVAS & \# ENCUESTADOS & PORCENTAJES \\
\hline DESCONOCIMIENTO & 255 & $66 \%$ \\
DESCONFIANZA & 110 & $29 \%$ \\
INSEGURIDAD & 19 & $5 \%$
\end{tabular}

Tabla $N^{\circ}$ 4.- Nivel de conocimientos con respecto a la utilización de los valores negociables por partes de las PYMES

Análisis

Observamos que 7 de cada 10 individuos opinan que las empresas no utilizan los valores negociables por Desconocimiento mientras que 2 de cada 10 individuos piensan que no lo hacen por Desconfianza, la alternativa Inseguridad para esta pregunta corresponde al $0.5 \%$, por lo tanto no es relevante para el análisis.

\section{Interpretación}

De la información obtenida se puede interpretar que el $66 \%$ de las personas opinan que las empresas no invierten en bolsa de valores debido al desconocimiento mientras el $29 \%$ no lo hace debido a la desconfianza puesto que es lógico no confiar en lo que no se conoce. 
¿Considera usted que el uso deficiente de los mercados financieros se debe principalmente a la baja promoción y difusión de información por parte de los mismos?

\begin{tabular}{ccc} 
ALTERNATIVAS & \# ENCUESTADOS & PORCENTAJES \\
\hline TOTALMENTE DE & 201 & $71 \%$ \\
ACUERDO & 79 & $28 \%$ \\
DE ACUERDO & 4 & $1 \%$ \\
EN DESACUERDO & 4
\end{tabular}

Tabla $N^{\circ}$ 5.- Nivel de conocimientos con respecto al uso de los mercados financieros

Análisis

Observamos que 7 de cada 10 individuos están Totalmente de acuerdo mientras que 2 están De acuerdo, la alternativa en Desacuerdo para esta pregunta corresponde al 0.1\%, por lo tanto es insignificante para el análisis.

\section{Interpretación}

De la información obtenida se puede interpretar que el $71 \%$ de la población encuestada está totalmente de acuerdo con que el deficiente uso de los mercados financieros se debe a que existe un bajo nivel de difusión de información proveniente de estos mismos mercados, mientras que el $28 \%$ está de acuerdo con la interrogante planteada. 
¿Conoce usted los mecanismos de financiamiento que la bolsa de valores ofrece tanto a las personas que tienen excesos de fondos disponibles, como las empresas que requieren capital?

\begin{tabular}{ccc} 
ALTERNATIVAS & \# ENCUESTADOS & PORCENTAJES \\
\hline SI & 102 & $27 \%$ \\
NO & 282 & $73 \%$
\end{tabular}

Tabla $N^{\circ}$ 6.- Nivel de conocimientos con respecto a los mecanismos de financiamiento que ofrece la bolsa de valores ecuatoriana

Análisis

Observamos que 7 de cada 10 individuos No conoce los mecanismos de financiamiento, mientras que 3 Si tiene conocimientos sobre estos.

Interpretación

De la información recabada podemos interpretar que el $73 \%$ de los encuestados no conoce los mecanismos de financiamiento que la bolsa de valores ofrece tanto a las personas que tienen excesos de fondos disponibles como a las personas que requieren capital por lo tanto, mientras que el $27 \%$ si tiene conocimientos de como poder invertir en bolsa de valores.

¿Cuál es la fuente de fondos externos más importante que usaría para financiar sus proyectos?

\begin{tabular}{ccc} 
ALTERNATIVAS & \# ENCUESTADOS & PORCENTAJES \\
\hline BANCOS & 266 & $69 \%$ \\
COOP. DE AHORRO Y & 84 & $22 \%$ \\
CREDITO & & $9 \%$ \\
BOLSA DE VALORES & 34 & $0 \%$ \\
USUREROS & 0 &
\end{tabular}

Tabla $N^{\circ}$ 7.- Nivel de conocimientos con respecto a la importancia de la utilización de fondos externos para financiar proyectos 
Análisis

Observamos que 7 de cada 10 individuos utilizarían los Bancos para financiar sus proyectos mientras que 2 de cada 10 utilizarían las Cooperativas, las alternativas Bolsa de valores y Usureros para esta pregunta corresponde al $0.9 \%$ y $0 \%$, por lo tanto no son relevantes para el análisis.

\section{Interpretación}

De la información recabada podemos interpretar que el $69 \%$ de los encuestados piensan que la mejor opción de fondos externos para financiar sus proyectos son los bancos mientras que el $22 \%$ optarían por las cooperativas de ahorro y crédito.

Si los ingresos que usted percibe le permitieran generar algún tipo de ahorro. ¿Estaría usted dispuesto a invertir en la bolsa de valores?

\begin{tabular}{ccc} 
ALTERNATIVAS & \# ENCUESTADOS & PORCENTAJES \\
\hline SI & 164 & $43 \%$ \\
NO & 25 & $6 \%$ \\
TAL VEZ & 195 & $51 \%$
\end{tabular}

Tabla $N^{\circ}$ 8. - Nivel de conocimientos con respecto a la disposición que tienen los habitantes de la provincia del guayas de invertir en la bolsa de valores

Análisis

Observamos que de cada 10 individuos 5 Tal vez estarían dispuestos a invertir en la bolsa de valores, 4 Si lo harían mientras que solo 1 No estaría dispuesto a invertir en bolsa de valores. 


\section{Interpretación}

De la información obtenida podemos interpretar que el $43 \%$ de los encuestados están dispuestos a invertir en la bolsa de valores dependiendo de los ingresos que perciben, mientras que la mayor parte de los encuestados con un $51 \%$ no están claros en su decisión.

\section{Conclusiones.}

Después de haber analizado los resultados obtenidos en la presente investigación he llegado a las siguientes conclusiones:

El mercados de valores es considerado como el mercado más importante a nivel mundial ya que es uno de los mayores centros de conexión entre inversionistas y ahorradores, razón por la cual tienen transcendental efecto en las economías de cualquier país puesto que al incrementar la inversión se reduce la salida de capitales, se impulsa la producción interna y como resultado se disminuyen los niveles de desempleo e incrementa el PIB.

Sin duda alguna la inversión y el financiamiento a través de la bolsa de valores ofrecen mayor rentabilidad y mejores condiciones que el sistema bancario tanto en plazos y reducción de costos para el sector empresarial, así como mejores rendimientos por sus ahorros para los inversionistas.

La creación de un manual básico de inversión contribuirá al fortalecimiento e incremento de los conocimientos en temas bursátiles de la población guayasense. 
Dentro de la economía ecuatoriana el sector productivo es considerado como uno de los más preponderantes, por lo tanto es necesario que el mercado de valores se dirija a este con mayor concentración, dándoles a conocer nuevas alternativas de financiamiento.

\section{Bibliografía.}

Díaz Mata, A. (2005). Una propuesta para un sistema de análisis técnico para compra y venta de acciones en la Bolsa Mexicana de Valores. Contaduría y Administración, 215(1), 25-48.

Garay Anaya, G. (2015). Las Finanzas Conductuales, el Alfabetismo Financiero y su Impacto en la Toma de Decisiones Financieras, el Bienestar Económico y la Felicidad. PERSPECTIVAS, 36(1), 7-34.

Moreno García, E., Vázquez Cotera, D., Hernández Mejía, S., \& Larios Ojeda, L. (2015). Interdependencia de los mercados de valores en el mundo. Economía: Teoría y práctica, 43(1), 155-181.

Paresh Kumar, N., Sagarika, M., \& Seema, N. (2011). Do market capitalization and stocks traded converge? New global evidence. Journal of Banking \& Finance, 35(10), 2771-2781.

Peña, I. (1991). Sobre la relación entre los mercados bursátiles internacionales y la bolsa de Madrid. Madrid: Universidad Carlos III.

Pico Pico, G., \& Pulgar León, A. (2006). Gestión Financiera y Participación en el Mercado de Capitales a través del Asesor de Inversiones. Actualidad Contable FACES, 9(13), 128-138. 